\title{
1 A preclinical biosensor for detecting phenylalanine photometrically \\ 2 in plasma and whole blood samples
}

3 Paul Eduardo David Soto Rodriguez ${ }^{a * *}$, Morgane Valles ${ }^{\mathrm{a}}$, Agostino Romeo ${ }^{\mathrm{a}}$, Rafael

$4 \quad$ Artuch $^{* * b}$, Samuel Sánchez***a,c.

5

6

7

8

9

10

11

12
${ }^{a}$ Institute for Bioengineering of Catalonia, Barcelona, Spain.

${ }^{b}$ Clinical Biochemistry Department, Institut de Recerca Sant Joan de Déu and CIBERER, , Barcelona, Spain.

'Institució Catalana de Recerca i Estudis Avançats (ICREA), Pg. Lluís Companys 23, 08010 Barcelona, Spain

${ }^{\#}$ Current address: Aix-Marseille University \& Institute of Biosciences and Biotechnologies, CEA Cadarache, France.

*Corresponding author : p.e.d.soto.rodriguez@gmail.com

**Corresponding author: $\underline{\text { Rartuch@sjdhospitalbarcelona.org }}$

***Corresponding author : ssanchez@ibecbarcelona.eu 
ABSTRACT.

17 Phenylketonuria (PKU) is a metabolic disease resulting from a deficiency in the enzyme 18 phenylalanine hydroxylase, increasing L-Phenylalanine (L-Phe) values in the blood and 19 consequently in the brain. If untreated, PKU leads to neurological damage, which can be prevented by following a diet low in L-Phe. Thus, early detection of PKU in newborns is essential. The disease's screening and monitoring are centralized in reference centers, which require specialized equipment. However, using these techniques, sample treatment is required before the analysis, and trained personnel must perform and interpret the results. In this work, we present an enzyme-based-photometric strategy to measure blood L-Phe. An enzymatic mixture, selective for L-Phe, is immobilized on an UV transparent well, and the amount of consumed cofactor is monitored at $340 \mathrm{~nm}$. Standard plasma and whole blood samples were chosen to prevalidate the sensor. The samples were spiked with an increasing amount of L-Phe, accurately discriminating between physiological and pathological L-Phe concentrations. The strategy can be easily extended to analyzing other samples, such as urine or sweat. The proposed photometric system allows to analyze up to 16 samples simultaneously within a matter of hours. The measurements are relatively fast, versatile, cost-effective, and easy to carry out.

Keywords: Phenylketonuria; L-phenylalanine; photometric biosensor; phenylalanine dehydrogenase. 


\section{Introduction}

Phenylketonuria (PKU) is a genetic metabolic disease caused by a deficiency of the hepatic enzyme phenylalanine hydroxylase (PAH), leading to high blood L-Phenylalanine (L-Phe) levels, which can cross the blood-brain barrier and accumulate in the brain. This phenomenon has been related to different pathomechanisms. Among others, white matter structural changes have been demonstrated in PKU. Also, high L-Phe levels have been correlated to low availability of neurotransmitter precursors (tyrosine and tryptophan) in the central nervous system, leading to decreased biosynthesis of dopamine and serotonin(Bilder et al. 2016). The main consequences of having high brain L-Phe levels for long periods are that they induce IQ loss, lead to cognitive and attention issues and behavior problems, among other neurological complications.(Romani et al. 2017) Implementation of a specialized diet during the newborn period averts the complications associate to the disease.

PKU has a varying prevalence worldwide, with a mean of $0.01 \%$ in the US and Europe, higher incidences in countries such as Turkey and Ireland, and lower in Finland.(Paul and Brosco 2013) The current neonatal screening approach(Paul and Brosco 2013; van Wegberg et al. 2017) generally consists of using tandem mass spectrometry (MS/MS) to detect, among other diseases, all potential cases related to high levels of L-Phe, which include those with PAH deficiency (the most common cause of hyperphenylalanemia), those with tetrahydrobiopterin $\left(\mathrm{BH}_{4}\right)$ metabolism defects, and those with the recently reported hyperphenylalaninemia due to mutation at the DNAJC12 gene. This neonatal screening test is done on dried blood on filter paper specimens with a L-Phe cut-off value of $120 \mu \mathrm{mol} / \mathrm{L}$, and are performed, like most neonatal screening tests during the 24-48 hours of life. These initial screening results are communicated to the newborn's family during the first week of life, after which a definitive differential diagnosis of the different diseases leading to hyperphenylalaninemia is (ideally) given during the first month of life. The routine application of next-generation sequencing (NGS) techniques is accelerating this process. While this early diagnostic activity is fully implemented in developed countries through national screening programs, the same is not standard practice in most developing countries. Therefore, there is an unmet need for an alternative, more affordable analytical L-Phe detection strategy in developing countries.

Once PKU has been detected and diagnosed, L-Phe levels must be continuously monitored for life during the imposed low L-Phe diet therapy, which is the most common treatment against PKU. Routine phenylalanine monitoring depends on a patient's age, varying from weekly frequency in infants to fortnightly or monthly frequency in children $<12$ years of age and adolescents/adults. Regarding recommended L-Phe values for follow-up checks, they vary slightly between American and European guidelines. However, it is generally agreed that L-Phe values below $360 \mu \mathrm{mol} / \mathrm{L}$ for children less than 12 years of age and women before conception and pregnancy, or below $600 \mathrm{umol} / \mathrm{L}$ for adolescents/adults, are considered safe (European guidelines(Lowe, DeLuca, and Arnold 2020)). Furthermore, L-Phe being an essential amino acid for average growth and development, values $<120 \mu \mathrm{mol} / \mathrm{L}$ are not recommended for PKU patient in long-term follow-ups. Alternative therapy to the low L-Phe diet consists of administering $\mathrm{BH}_{4}$ (on behalf of the International Working Group on Neurotransmitter related Disorders (iNTD) et al. 2020), a PAH co-factor, to patients who are candidates for this therapy. However, only a tiny percentage of PKU cases have shown to respond positively to this treatment. A few alternative therapies have recently received approval by the Food and Drug Administration (FDA) and show promise for improving the quality of life of PKU patients. The first FDA-approved (on May 24, 2018) enzyme substitution therapy for (adult) patients with PKU 
is Pegvaliase, a pegylated Phenylalanine ammonia-lyase (PAL) enzyme that converts phenylalanine to ammonia and trans-cinnamic acid. This therapy is currently on the market under the brand name "Palynziq" (Biomarin) and is recommended for patients with documented blood L-Phe $>600 \mu \mathrm{mol} / \mathrm{L}$ who have failed existing management strategies. However, the administration of Pegvaliase does come with a risk of anaphylaxis and is therefore available through the Risk Evaluation and Mitigation Strategy (REMS) program(Hydery and Coppenrath 2019). Other therapeutic approaches for PKU are currently under investigation(Charbonneau et al. 2020) and may be found at an available online database (e.g. www.clinicaltrials.gov).

Many studies aimed at developing new PKU monitoring strategies, relying on several different approaches, have been published. These include enzyme-based(Gubica et al. 2015; Mangombo et al. 2013; Naghib, Rabiee, Omidinia, and Khoshkenar 2012; Naghib, Rabiee, Omidinia, Khoshkenara, et al. 2012; Naghib, Rabiee, and Omidinia 2014a, 2014b; Omidinia, Khanehzar, et al. 2013; Omidinia, Shadjou, and Hasanzadeh 2014b; Villalonga et al. 2007, 2008; Weiss et al. 2007; Zhuo Wang et al. 2005), aptamer-based (Idili et al. 2021), (Omidinia, Shadjou, and Hasanzadeh 2014a) and nanomaterials(Hasanzadeh et al. 2009, 2012; Hasanzadeh, Shadjou, and Omidinia 2013; Omidinia, Shadjou, and Hasanzadeh 2013; Wu et al. 2017) based electrochemical sensors, as well as immune sensors,(Kubota, Mizukoshi, and Miyano 2013) quartz crystal microbalance (QCM),(Cho et al. 2017; Emir Diltemiz et al. 2017; Mirmohseni, Shojaei, and Farbodi 2008) extended field gate field effect transistor(Iskierko et al. 2017) and photometricbased strategies. Some of the principle challenges facing the development of an effective biosensor for PKU monitoring include (1) having a linear range which covers the $120 \mu \mathrm{mol} / \mathrm{L}$ and $360 \mu \mathrm{mol} / \mathrm{L}$ key threshold values for L-Phe described previously, (2) being able to detect L-Phe in patient-derived samples with low chemical interference (e.g. from other amino acids), (3) a design that is intuitive and user-friendly and (4) a fast detection time.

Despite the broad interest, an easy, low-cost and fast L-Phe analytical system has yet to be developed. In this work, we provide such a method based on the indirect photometric detection of L-Phe, which uses a commercially available phenylalanine dehydrogenase (PDH) enzyme. We chose PDH over PAL to adapt our biosensor system to detect analytes using other dehydrogenases (Lactate-, tyrosine-, glucose-, pyruvate-, etc dehydrogenase). The photometric detection system was designed to be compatible with UV-transparent microplates. The PDH enzyme, mixed with stabilizing components, is immobilized at the bottom of the microplate well using a Nafion membrane. This strategy minimizes the preparation for the final end-user as the modified microplate can easily be stored at $4^{\circ} \mathrm{C}$ and used when measurements are needed. The end-user only has to prepare the measurement solution containing the sample, buffer and cofactor. The photometric system described in this work was fully characterized, with the best working conditions, long-term storage, the study of possible interferents and initial validation in plasma samples (the sensor output correlates linearly with the values of the hospital, determined using a validated protocol) and whole blood samples. The results described here offer a promising solution for PKU L-Phe monitoring. 
All chemicals were used as received without any further purification. Glycine Buffer $\mathrm{pH} 10.5$ (prepared with Glycine ReagentPlus, $\geq 99 \%$ (HPLC); Sigma Aldrich), phosphate buffer (PB) pH 7.4 ( $\mathrm{pH}$ adjusted with Potassium Phosphate monobasic ACS reagent $\geq 99 \%$, Sigma Aldrich), L-Phe BioUltra $\geq 99 \%$ (NT); Sigma Aldrich, $\beta-N A D$; Thermo Fischer Scientific, L-Phenylalanine Dehydrogenase from Sporosarcina sp. (PDH, $50 \mathrm{U}$ ); Sigma Aldrich, Bovine Serum Albumin (BSA) lyophilized powder; Sigma Aldrich, 0.5\% Nafion 117 solution; Sigma Aldrich. UV transparent microplates were used. The SYNERGY HTX multi-mode microplate reader (Biotek) was used for the absorption measurements.

Plasma and whole blood samples were provided by the Hospital "San Joan de Deu", the reference center for PKU follow-up in Catalonia. Both plasma and whole blood samples were prepared from quality control materials and healthy volunteers, spiked with different amounts of L-Phenylalanine ( $n=106$ in total for plasma; 61 samples were used to establish the optimal technical conditions of the biosensor, and then, the remaining 45 were used to be compared with those analyzed in HSJD and $n=5$ for whole blood). The range of concentrations was between $49-1800 \mu \mathrm{M}$, covering the range of standard and very high phenylalanine values. Lphenylalanine values were determined by a fully validated reference method (liquid chromatography/tandem mass spectrometry) as previously reported.(Casado et al. 2018) This method has been accredited by the Spanish accreditation agency (ENAC, ISO15189 norm), and our laboratory participates in the external quality control scheme ERNDIM for amino acids (data available on request). For three L-Phe values $(300 \mu \mathrm{M}, 450 \mu \mathrm{M}$ and $700 \mu \mathrm{M})$, ten additional measurements in triplicate were performed ( 30 samples analyzed as a whole), and subsequently analyzed employing an f-test and a two-tailed student $t$-test to search for statistical differences among these groups. For the initial validation of the biosensor, all samples were analyzed in a blinded way. This study was approved by the Ethical Committee of Hospital Sant Joan de Déu (project number DTS18/00075). All methods were carried out in accordance with the 2013 revised Helsinki Declaration of 1964. Blood donor volunteers were recruited from Hospital Sant Joan de Déu laboratory staff. Informed consent was obtained from all subjects, and informed consent is available on request.

Dropcasted solution.

Prior to modifying the biosensor microplates, a solution of phosphate buffer $(\mathrm{pH} 7.4)$ containing BSA ( $20 \% \mathrm{~V} / \mathrm{V}), \mathrm{PDH}(8.4 \% \mathrm{~V} / \mathrm{V})$ and $0.5 \%$ of Nafion $(2.7 \% \mathrm{~V} / \mathrm{V})$ was prepared. An amount of $7 \mu \mathrm{L}$ of this solution was then drop cast to each test well, which constituted 45 wells of the $96-$ well plate, and left to dry overnight at $4^{\circ} \mathrm{C}$. The other wells in the plate were left unmodified to measure the blank negative controls. For initial measurements in glycine buffer, the final concentrations of BSA and PDH were $14 \mu \mathrm{M}$ and $1 \mathrm{U} / \mathrm{cm}^{2}$, respectively. In plasma, BSA and PDH concentrations were optimized to $28 \mu \mathrm{M}$ and $3 \mathrm{U} / \mathrm{cm}^{2}$, respectively (vide infra). For measurements performed in whole blood, the PDH concentration was increased to $6 \mathrm{U} / \mathrm{cm}^{2}$, while BSA concentration remained fixed at $28 \mu \mathrm{M}$.

\section{Measurement protocol.}

For the measurements conducted in buffer, $200 \mu \mathrm{L}$ of $250 \mathrm{mM}$ glycine buffer (pH 10.5), with $6 \mathrm{mM} \mathrm{NAD}^{+}$and variable concentrations of L-Phe were added to each of the blank wells. After running the blank control measurements, $180 \mu \mathrm{L}$ of this measurement buffer were added to the test wells. For measurements performed in plasma, the L-Phe was omitted from the measurement buffer, and $5 \mu \mathrm{L}$ of the plasma samples were added to the same buffer. Blank 
controls and test experiments were performed using the same procedure as those in the buffer. For measurements in whole blood, $1 \mu \mathrm{L}$ of a $1000 \mathrm{X}$ dilution of the whole blood samples were added to the measurement buffer, which contained $6 \mathrm{mM} \mathrm{NAD}^{+}$. Measurements were then conducted as previously described.

\section{Sensor preparation.}

The optical biosensor was fabricated according to the steps laid out in Figure $1 \mathrm{~A}$. First, the wells were modified with the PDH enzyme, mixed in a solution along with BSA and Nafion (see Figure $1 \mathrm{~B})$, dropcasted into the wells and left to dry. BSA was added to the mixture to stabilize PDH, and the Nafion polymer served to fix the enzyme mixture onto the bottom of the well, limiting desorption. PDH activity was then assayed by adding a solution of $\mathrm{NAD}^{+}$and L-Phe dissolved in glycine buffer at $\mathrm{pH} 10.5$, and measuring the rate of change in absorbance at $340 \mathrm{~nm}$ over time, corresponding to the absorbance peak of reduced co-factor, NADH (see the corresponding chemical reaction in Figure $1 \mathrm{D}$ ). Indeed, the interconversion of $\mathrm{NAD}^{+}$with NADH is a commonly used technique to evaluate the catalytic activity of dehydrogenases, as the UV absorption spectra of the reduced form differ from that of the oxidized form by the presence of a second peak at $340 \mathrm{~nm}$ in the UV range (Gloster and Harris 1962). For a fixed concentration of NAD', the initial slope of the $\mathrm{Abs}_{340}$ response over time increased with increasing L-Phe concentration.

(insert Figure 1 here)

\section{Statistical analysis}

The statistical analysis was done by initially performing a F-test to evaluate if equal or unequal variances need to be considered for the t-test analysis. Once it was established that both populations presented equal variances, a two-tail t-test was performed considering a cut-off $p$ value of 0.05 , rejecting the null hypothesis that both populations have no significant difference (presenting a mean difference of zero) when below this value.

\section{Bill of material (BOM)}

The total cost is provided for measurements in the whole blood. We recommend peforming triplicates of the measurements for a more reliable output. Therefore, the cost per patient is considered from the cost of the measurement done in triplicate.

The cost per patient is calculated as follows: we consider the cost of the amount of enzyme per well $0.0196 \mathrm{U} /$ well (PDH $437 €$ for $50 \mathrm{U}$ ), which gives $0.1713 € /$ well which gives $8.22 € /$ microplate (48 wells of the test). The amount of co-factor NAD $(72.2 \mathrm{E} / \mathrm{g}$ ) we use is $42.12 \mathrm{mg} / \mathrm{microplate}$, giving $2.97 € /$ microplate. For the BSA (50g costs $181.8 €$ ) we make $24 \mathrm{mg} / 90 \mathrm{ul}$ and use 18 $\mathrm{uL} /$ well, giving thus $0.84 € /$ microplate. The Nafion $(25 \mathrm{ml} / 176.5 €)$ is diluted $10 \mathrm{X}$, and $18 \mathrm{ul}$ is added, which corresponds to $0.608 € /$ microplate. The final estimate is $13.64 € /$ microplate, including the price of the microplate itself, which corresponds to $0.28 € /$ measurement $(0.85 €$ in triplicate). For the optimized conditions in plasma, we use 2X BSA $(2 \times 0.84 €)+3 \mathrm{XPDH}(3 \times 8.22 €)$ more. The described calculation gives $30.02 €$ per microplate, $0.64 €$ per measurements and $1.92 €$ per triplicate and therefore per patient. For whole blood, we use 2X BSA ( $2 \times 0.84 €)+9 \mathrm{X}$ $\mathrm{PDH}(3 \times 8.22 €)$ more and therefore, the price per patient is $4.95 €$. 
The sensor is characterized by varying many parameters to find the optimal working conditions of the PKU biosensor (Figure S1). The optimization considers high-performance and costefficiency. For the initial characterization, we used a fixed concentration PDH $1 \mathrm{U} / \mathrm{cm}^{2}$., BSA 14 $\mu \mathrm{M}, \mathrm{NAD}{ }^{+} 6 \mathrm{mM}$ and $\mathrm{T}=30^{\circ} \mathrm{C}$ and $\mathrm{pH}=10.5$. Each variable was varied independently, see Figure S1.

212 First, for the dropcasting mixture used to modify the wells, it was found that the signal output was highest at the initial $\mathrm{pH}$ of 10.5 (Figure $\mathrm{S} 1 \mathrm{~A}$ ), then $\mathrm{NAD}^{+}$was varied, and $6 \mathrm{mM}$ was chosen (Figure S1 B). The best temperature was also shown to be $30^{\circ} \mathrm{C}$ (Figure S1 C). These were the conditions used and chosen to do the initial calibration tests and interference analysis in the buffer. For plasma analysis, the BSA and PDH amount were optimized (Figure S1 D) to avoid any matrix effect when performing tests on plasma. This optimization showed that $3 \mathrm{U} / \mathrm{cm}^{2}$ of PDH needed to be immobilized in each well to provide the best performance. BSA was added to stabilize $\mathrm{PDH}$, preventing it from denaturing during the immobilization process. Upon increase of BSA concentration in the dropcasting mixture, the overall activity of PDH was improved (Figure S1 D), and was optimal at $28 \mu \mathrm{M}$.

To characterize the enzymatic activity of the biosensor in the newly optimized conditions, the initial slopes from the biosensor assays, conducted at different concentrations of L-Phe (in buffer), were converted to the amount of generated NADH per second using Beer-Lambert Law and fitted with a Michaelis-Menten equation (Figure $2 \mathrm{~A}$ ). An apparent $K_{\mathrm{M}}$ of 1,683.021 $\mu \mathrm{M}$ was obtained from the Michaelis-Menten fit, and the reaction rate of the biosensor reached saturation for approximately $5 \mathrm{mM}$ of L-Phe substrate, a value outside the physiological and pathological ranges. Figure $2 \mathrm{~B}$ shows the linear range of the Michaelis-Menten graph in Figure $2 \mathrm{~A}$, which is between $30 \mu \mathrm{M}$ and $1 \mathrm{mM}$. Good linearity was observed $\left(R^{2}=0.961\right)$ covering both the safe range $(<360 \mu \mathrm{mol} / L)$ and those corresponding to mild to severe hyperphenylalaninemia (from 360 to higher than $1000 \mu \mathrm{mol} / \mathrm{L}$ ), demonstrating the potential adequateness of the photometric biosensor for PKU monitoring. Moreover, the sensitivity and limit of detection (LOD) of the biosensor could be determined from the linear fit of the data in Figure 2 B (5.86 $x 10^{-5} \mathrm{Abs}^{-1} \mathrm{~s}^{-1}$ and $1.03 \mu \mathrm{M}$, respectively). The LOD and additional parameters of interest are indicated in Table 1, which also compares and contrasts the results from the biosensor described in this work with similar devices reported in the literature.

(insert Figure $2+$ Table 1)

The selectivity and shelf-life of the biosensor are evaluated employing the initial conditions. PDH's specific reactivity towards L-Phe is often hindered by the presence of interferents, such as tyrosine (see Table 1). Therefore, the PKU biosensor's activity towards several different (potentially interferent) amino acids was tested (Figure $2 \mathrm{C}$ ). The PKU biosensor presented high selectivity to the L-Phe substrate, with minimal interference from arginine, glutamine, tryptophan, cysteine and tyrosine (Figure $2 \mathrm{C}$ ). Each interferent was tested at relevant physiological concentrations present in plasma(Canepa et al. 2002), bearing in mind that these reference values may vary slightly among laboratories. The stability of the biosensor over time is displayed in the inset of Figure $2 \mathrm{C}$, where it is shown that, if stored at $4{ }^{\circ} \mathrm{C}$, the biosensing capability is stable over the period of 4 weeks.

The PKU biosensor was thus optimized for L-Phe quantification in $\mathrm{pH} 10.5$ buffer and could undergo validation on anonymous plasma samples in a mixture containing quality control materials at different concentrations, provided by the Hospital "San Joan de Deu" (HSJD). As 
previously indicated, to avoid any matrix effect from the plasma samples, the measuring conditions were adjusted. The PKU biosensor was operated using the following optimized parameters in the buffer: $6 \mathrm{mM} \mathrm{NAD}^{+}, 30^{\circ} \mathrm{C}, \mathrm{pH} 10.5,3 \mathrm{U} / \mathrm{cm}^{2}$ of PDH and $28 \mu \mathrm{M}$ of BSA. We also reduced the sampling volume to $2.5 \% \mathrm{v} / \mathrm{v}$, which again helps avoid any matrix effect, see methods section. No effect from possible interferents was observed under these conditions; see Figure 2D. A single-blind test with our L-Phe biosensor on the plasma samples was then carried out, with no prior knowledge of the samples' L-Phe concentration, determined by the hospital using MS/MS. In Figure $3 \mathrm{~A}$, a clear linear correlation is found between our biosensor output values with the hospital values provided at a later stage, supporting the robustness of our biosensor and providing a calibration curve that allows converting the units from NADH/s to $\mu \mathrm{M}$. In Figure 3B, by applying the correlation described in Figure 3A, the biosensor was used to detect a range of L-Phe concentrations and classify them into healthy (green), mildly elevated (orange), high (red) and significantly elevated (burgundy) groups by using corresponding threshold values of [L-Phe] $<120 \mu \mathrm{M}, 120 \mu \mathrm{M}<$ [L-Phe] $<360 \mu \mathrm{M}, 360 \mu \mathrm{M}<$ [L-Phe] $<1200 \mu \mathrm{M}$ and $1200 \mu \mathrm{M}<$ [L-Phe], respectively.

The current preclinical study is the first stepping stone towards a full clinical validation of the proposed biosensor in the hospital setting. These results enable our biosensor to be potentially used for monitoring the L-Phe levels of pre-diagnosed PKU patients. Considering that L-Phe values below $360 \mu \mathrm{M}$ are considered safe for children younger than 12 years old and that those above $600 \mu \mathrm{M}$ are higher than those recommended for PKU patients older than 12 years, we included a plot of measurements done on 30 plasma samples, measured in triplicate, 10 with $300 \mu \mathrm{M}$ [L-Phe], 10 with $450 \mu \mathrm{M}$ [L-Phe], and 10 with $700 \mu \mathrm{M}$ [L-Phe] (Figure 4A). The confidence interval for these measurements, was obtained by performing a $t$-test analysis. As can be observed, the calculated $p$ values are below $p=0.05$ and therefore rejects the null hypothesis (mean difference between both populations is zero) and therefore indicating that the $300 \mu \mathrm{M}$, $450 \mu \mathrm{M}$ and $700 \mu \mathrm{M}$ [L-Phe] are statistically distinguishable between each other. The presented data shows that the device can alert when L-Phe concentration values are unsafe, and by extension when the diet or $\mathrm{BH}_{4}$ therapies need to be readjusted.

To complete our preclinical study, the biosensor was tested against whole blood samples. In this case, to achieve a good sensing performance of the L-Phe biosensor, an even more significant dilution was required (5000 times more diluted compared to the blood plasma) and an increase in enzyme concentration $\left(9 \mathrm{U} / \mathrm{cm}^{2}\right)$. A total of five anonymous whole blood samples were blind tested in a similar way to the tests done on plasma. The obtained correlation function is presented in Figure 4B.

Overall, a highly sensitive and stable L-Phe photometric biosensor for plasma samples is described.

The overview presented in Table 1 shows that our proposed photometric setup has many benefits over others reported in the literature. Our PKU sensor is easily scalable, cost-effective, and could analyze L-Phe in different biological samples if needed. Moreover, multi-analyte detection would be possible through the immobilization of other dehydrogenases (e.g. glucose - glucose-6-phosphate dehydrogenase, aldehyde - Aldehyde dehydrogenases, etc.), and the measurements are relatively fast compared to traditional methods. The system is simple, easy to prepare (minimum processing is needed), has long-term stability (only ref(Pijanowska and Remiszewska 2006)presents results for the durability of their sensor) and is fully characterized. 
The L-Phe biosensor has been pre-validated on plasma and whole blood samples provided and analyzed by the HSJD. The obtained results show that the presented sensor design is highly recommended for determining L-Phe levels in blood plasma (only a few work showed results in the same matrix previously (Brunhuber et al. 2000; Hummel, Schütte, and Kula 1988; Johnson and Morrison 1970). Our preliminary data also shows the possibility of adapting the sensor to measure L-Phe in whole blood (only (Arakawa et al. 2011) in Table 1 describes a similar capacity) potentially allowing an efficient L-Phe monitoring system for use in at-home settings or outside reference centers.

To highlight the potential of our biosensor and to strengthen the data shown in Figure 3, 30 additional blood plasma sample measurements were done under the same conditions (i.e blind measurement and posterior validation with the hospital values), with the results shown in the plot of the supporting information in Figure S2. Significant statistical differences were observed among the different concentrations chosen, supporting the future usefulness of the biosensor, once fully validate, to make clinical decisions regarding treatment modification.

The results on whole blood samples are of great importance, as measuring whole blood samples is not straightforward due to the high complexity of this sample. Moreover, this provides an excellent alternative to pre-existing diagnostic L-Phe analysis systems. However, it should be noted that the need to increase the enzyme concentration raises the overall cost of the biosensor considerably concerning the tests on plasma. Strategies to mitigate this increase in the price of the biosensor while improving its sensitivity to L-Phe in whole blood will be further explored in future work. Nevertheless, the cost of the test per patient on blood plasma is below 2 euros and for whole blood samples remains below 5 euros (see the methods section for a detailed bill of materials), which makes our sensor a desirable option for developing countries.

\section{Limitations and future directions.}

This is a preclinical study, the results of which need to be thoroughly tested employing a "validation of a medical device" study. The first step will be to fabricate a user-friendly point-ofcare device used in the hospital laboratory, which should be compared with the standard method for plasma and whole blood L-Phe monitoring during PKU patient follow-up. The system needs to be robust since the next step will be designing an at-home device for L-Phe monitoring. As previously mentioned, according to the PKU guidelines, L-Phe monitoring frequency varies between weekly and monthly, depending on the patient's age. The possibility of analyzing L-Phe values at home with a higher frequency (as occurs in other diseases, such as diabetes) can improve our knowledge about the clinical outcome of PKU patients concerning the fluctuation of blood L-Phe over time. 
332 Overall, a highly sensitive and stable L-Phe photometric biosensor for plasma is described. Its 333 fast measurement and analysis can allow in the future, after extensive clinical validation, for more frequent analyses, which is crucial for PKU monitoring and understanding the fluctuations of L-Phe levels over time. The device is potentially easily adaptable for analyzing other biological fluids (urine or sweat), thus reducing invasiveness. The immobilization of the enzyme at the bottom of the microplate wells is highly convenient and provides excellent versatility to other enzymatic reactions using $\mathrm{NAD}^{+}$as a co-factor (namely dehydrogenases). This could lead to multi-biosensing photometric platforms, on the same microplate, by parallel analysis of the absorbance kinetics at $340 \mathrm{~nm}$.

\section{Acknowledgements}

The research leading to these results was achieved thanks to the financial support from the for European Research Council (ERC) under the European Union's Horizon 2020 research and innovation programme (grant agreement No. 790163) (LABPATCH) and Juan de la Cierva "formación" program $2016 \mathrm{FJCl}$-2016-29512. Financial support was also provided by the European Commission under Horizon 2020's Marie Skłodowska-Curie Actions COFUND scheme [Grant Agreement no.712754] and by the Severo Ochoa programme of the Spanish Ministry of Economy and Competitiveness [Grant SEV-2014-0425(2015-2019)].

\section{Author contribution statement}

PEDSR, AR, and SS conceived the idea, designed and lead the experiments. PEDSR, MV and AR did the characterization on the buffer. PEDSR and MV realized the experiments and optimization for the detection of plasma and whole blood samples. RA collected and instructed on the correct manipulation of the hospital provided samples. All authors analyzed and discussed the data. All authors contributed to the paper writing and agreed with the final version.

\section{References}

Arakawa, Takahiro, Tomoyuki Koshida, Tomoko Gessei, Kumiko Miyajima, Daishi Takahashi, Hiroyuki Kudo, Kazuyoshi Yano, and Kohji Mitsubayashi. 2011. "Biosensor for LPhenylalanine Based on the Optical Detection of NADH Using a UV Light Emitting Diode." Microchimica Acta 173(1-2):199-205. doi: 10.1007/s00604-010-0536-5.

Bilder, Deborah A., J. Kay Noel, Erin R. Baker, William Irish, Yinpu Chen, Markus J. Merilainen, Suyash Prasad, and Barbara J. Winslow. 2016. "Systematic Review and Meta-Analysis of Neuropsychiatric Symptoms and Executive Functioning in Adults With Phenylketonuria." Developmental Neuropsychology 41(4):245-60. doi: 10.1080/87565641.2016.1243109.

Brunhuber, Norbert M. W., James B. Thoden, John S. Blanchard, and Janeen L. Vanhooke. 2000. "Rhodococcus L-Phenylalanine Dehydrogenase: Kinetics, Mechanism, and Structural Basis for Catalytic Specifity ${ }^{+}$, ‡." Biochemistry 39(31):9174-87. doi: 10.1021/bi000494c. 
Canepa, Alberto, José Carolino Divino Filho, Alberto Gutierrez, Alba Carrea, Ann-Marie Forsberg, Eva Nilsson, Enrico Verrina, Francesco Perfumo, and Jonas Bergström. 2002. "Free Amino Acids in Plasma, Red Blood Cells, Polymorphonuclear Leukocytes, and Muscle in Normal and Uraemic Children." Nephrology Dialysis Transplantation 17(3):413-21. doi: 10.1093/ndt/17.3.413.

Casado, Mercedes, Cristina Sierra, Marta Batllori, Rafael Artuch, and Aida Ormazabal. 2018. "A Targeted Metabolomic Procedure for Amino Acid Analysis in Different Biological Specimens by Ultra-High-Performance Liquid Chromatography-Tandem Mass Spectrometry." Metabolomics 14(6):76. doi: 10.1007/s11306-018-1374-4.

Charbonneau, Mark R., Vincent M. Isabella, Ning Li, and Caroline B. Kurtz. 2020. "Developing a New Class of Engineered Live Bacterial Therapeutics to Treat Human Diseases." Nature Communications 11(1):1738. doi: 10.1038/s41467-020-15508-1.

Cho, Sun-Min, Da-Seul Kim, Min-Hee Kim, and Seong-Ho Choi. 2017. "Fabrication of a 8 Cyclodextrin Modified Quartz Crystal Microbalance Biosensor and Its Chiral Recognition." Sensor Letters 15(3):261-65. doi: 10.1166/sl.2017.3790.

De Silva, Veronica, Charlie D. Oldham, and Sheldon W. May. 2010. "L-Phenylalanine Concentration in Blood of Phenylketonuria Patients: A Modified Enzyme Colorimetric Assay Compared with Amino Acid Analysis, Tandem Mass Spectrometry, and HPLC Methods." Clinical Chemistry and Laboratory Medicine 48(9). doi: 10.1515/cclm.2010.271.

Emir Diltemiz, Sibel, Rüstem Keçili, Arzu Ersöz, and Rıdvan Say. 2017. "Molecular Imprinting Technology in Quartz Crystal Microbalance (QCM) Sensors." Sensors 17(3):454. doi: $10.3390 / s 17030454$.

Gloster, J. A., and P. Harris. 1962. "Observations on an Enzymic Method for the Estimation of Pyruvate in Blood." Clinica Chimica Acta 7(2):206-11. doi: 10.1016/00098981(62)90011-6.

Gubica, Tomasz, Katarzyna Pałka, Łukasz Szeleszczuk, and Marianna Kańska. 2015. "Enhanced Enzymatic Activity of Phenylalanine Dehydrogenase Caused by Cyclodextrins." Journal of Molecular Catalysis B: Enzymatic 118:89-94. doi: 10.1016/j.molcatb.2015.05.004.

Hasanzadeh, Mohammad, Ghasem Karim-Nezhad, Nasrin Shadjou, Maryam Hajjizadeh, Balal Khalilzadeh, Lotfali Saghatforoush, Mohammad Hossein Abnosi, Ali Babaei, and Sohrab Ershad. 2009. "Cobalt Hydroxide Nanoparticles Modified Glassy Carbon Electrode as a Biosensor for Electrooxidation and Determination of Some Amino Acids." Analytical Biochemistry 389(2):130-37. doi: 10.1016/j.ab.2009.03.024.

Hasanzadeh, Mohammad, Nasrin Shadjou, Sue-Tsong Chen, and Peyman Sheikhzadeh. 2012. "MCM-41-NH2 as an Advanced Nanocatalyst for Electrooxidation and Determination of Amino Acids." Catalysis Communications 19:21-27. doi: 10.1016/j.catcom.2011.12.007.

Hasanzadeh, Mohammad, Nasrin Shadjou, and Eskandar Omidinia. 2013. "Mesoporous Silica (MCM-41)-Fe2O3 as a Novel Magnetic Nanosensor for Determination of Trace Amounts of Amino Acids." Colloids and Surfaces B: Biointerfaces 108:52-59. doi: 10.1016/j.colsurfb.2013.02.015. 
Hsu, Chung-Yi, Mei-Hwa Lee, James L. Thomas, Ching-Ping Shih, Tzu-Lin Hung, Thou-Jen Whang, and Hung-Yin Lin. 2015. "Optical Sensing of Phenylalanine in Urine via Extraction with Magnetic Molecularly Imprinted Poly(Ethylene-Co-Vinyl Alcohol) Nanoparticles." Nanotechnology 26(30):305502. doi: 10.1088/0957$4484 / 26 / 30 / 305502$.

Hummel, Werner, Horst Schütte, and M. R. Kula. 1988. "Enzymatic Determination of LPhenylalanine and Phenylpyruvate with I-Phenylalanine Dehydrogenase." Analytical Biochemistry 170(2):397-401. doi: 10.1016/0003-2697(88)90651-3.

Hydery, Tasmina, and Valerie Azzopardi Coppenrath. 2019. "A Comprehensive Review of Pegvaliase, an Enzyme Substitution Therapy for the Treatment of Phenylketonuria." Drug Target Insights 13:117739281985708. doi: 10.1177/1177392819857089.

Idili, Andrea, Julian Gerson, Tod Kippin, and Kevin W. Plaxco. 2021. "Seconds-Resolved, In Situ Measurements of Plasma Phenylalanine Disposition Kinetics in Living Rats." Analytical Chemistry 93(8):4023-32. doi: 10.1021/acs.analchem.0c05024.

Iskierko, Z., A. Checinska, P. S. Sharma, K. Golebiewska, K. Noworyta, P. Borowicz, K. Fronc, V. Bandi, F. D'Souza, and W. Kutner. 2017. "Molecularly Imprinted Polymer Based Extended-Gate Field-Effect Transistor Chemosensors for Phenylalanine Enantioselective Sensing." Journal of Materials Chemistry C 5(4):969-77. doi: 10.1039/C6TC03812C.

Johnson, S. L., and Diana L. Morrison. 1970. "The Alkaline Reaction of Nicotinamide Adenine Dinucleotide, a New Transient Intermediate." Journal of Biological Chemistry 245(17):4519-24. doi: 10.1016/S0021-9258(19)63821-4.

Kubota, Kazuyuki, Toshimi Mizukoshi, and Hiroshi Miyano. 2013. "A New Approach for Quantitative Analysis of L-Phenylalanine Using a Novel Semi-Sandwich Immunometric Assay." Analytical and Bioanalytical Chemistry 405(25):8093-8103. doi: 10.1007/s00216-013-7081-0.

Lowe, Tracy Brock, Jane DeLuca, and Georgianne L. Arnold. 2020. "Similarities and Differences in Key Diagnosis, Treatment, and Management Approaches for PAH Deficiency in the United States and Europe." Orphanet Journal of Rare Diseases 15(1):266. doi: 10.1186/s13023-020-01541-2.

Mangombo, Z. A., D. Key, E. I. Iwuoha, and P. G. L. Baker. 2013. “Development of LPhenylalanine Biosensor and Its Application in the Real Samples." Insciences Journal 123. doi: 10.5640/insc.030101.

Messina, M. A., C. Meli, S. Conoci, and S. Petralia. 2017. "A Facile Method for Urinary Phenylalanine Measurement on Paper-Based Lab-on-Chip for PKU Therapy Monitoring." Analyst 142(24):4629-32. doi: 10.1039/C7AN01115F.

Mirmohseni, Ablolreza, Maryam Shojaei, and Maryam Farbodi. 2008. "Application of a Quartz Crystal Nanobalance to the Molecularly Imprinted Recognition of Phenylalanine in Solution." Biotechnology and Bioprocess Engineering 13(5):592-97. doi: 10.1007/s12257-008-0028-1. 
Naghib, Seyed Morteza, Mohammad Rabiee, and Eskandar Omidinia. 2014a. "Electroanalytical Validation of a Novel Nanobiosensing Strategy and Direct Electrochemistry of Phenylalanine Dehydrogenase for Clinical Diagnostic Applications." 9:2301-15.

Naghib, Seyed Morteza, Mohammad Rabiee, and Eskandar Omidinia. 2014b. "Electrochemical Biosensor for L-Phenylalanine Based on a Gold Electrode Modified with Graphene Oxide Nanosheets and Chitosan." Int. J. Electrochem. Sci 9:2341-53.

Naghib, Seyed Morteza, Mohammad Rabiee, Eskandar Omidinia, and Payam Khoshkenar. 2012. "Investigation of a Biosensor Based on Phenylalanine Dehydrogenase Immobilized on a Polymer-Blend Film for Phenylketonuria Diagnosis." Electroanalysis 24(2):407-17. doi: 10.1002/elan.201100391.

Naghib, Seyed Morteza, Mohammad Rabiee, Eskandar Omidinia, P. Khoshkenara, and Darya Zeini. 2012. "Biofunctionalization of Dextran-Based Polymeric Film Surface through Enzyme Immobilization for Phenylalanine Determination." Int. J. Electrochem. Sci 7(1):120-35.

Omidinia, Eskandar, Ali Khanehzar, Nasrin Shadjou, and Hamid Mohamadi Shabaz. 2013. "Covalent Immobilization of Phenylalanine Dehydrogenase on GlutaraldehydeModified Poly (3,4-Ethylenedioxy) Thiophene: Poly(Styrenesulfonate)/ Polyvinyl Alcohol Conducting Polymer Composite Films for Electrochemical Detection of LPhenylalanine." 5(5):597-608.

Omidinia, Eskandar, Nasrin Shadjou, and Mohammad Hasanzadeh. 2013. “(Fe3O4)-Graphene Oxide as a Novel Magnetic Nanomaterial for Non-Enzymatic Determination of Phenylalanine." Materials Science and Engineering: C 33(8):4624-32. doi: 10.1016/j.msec.2013.07.023.

Omidinia, Eskandar, Nasrin Shadjou, and Mohammad Hasanzadeh. 2014a. "Aptamer-Based Biosensor for Detection of Phenylalanine at Physiological PH." Applied Biochemistry and Biotechnology 172(4):2070-80. doi: 10.1007/s12010-013-0656-6.

Omidinia, Eskandar, Nasrin Shadjou, and Mohammad Hasanzadeh. 2014b. "Electrochemical Nanobiosensing of Phenylalanine Using Phenylalanine Dehydrogenase Incorporated on Amino-Functionalized Mobile Crystalline Material-41." IEEE Sensors Journal 14(4):1081-88. doi: 10.1109/JSEN.2013.2292875.

on behalf of the International Working Group on Neurotransmitter related Disorders (iNTD), Thomas Opladen, Eduardo López-Laso, Elisenda Cortès-Saladelafont, Toni S. Pearson, H. Serap Sivri, Yilmaz Yildiz, Birgit Assmann, Manju A. Kurian, Vincenzo Leuzzi, Simon Heales, Simon Pope, Francesco Porta, Angeles García-Cazorla, Tomáš Honzík, Roser Pons, Luc Regal, Helly Goez, Rafael Artuch, Georg F. Hoffmann, Gabriella Horvath, Beat Thöny, Sabine Scholl-Bürgi, Alberto Burlina, Marcel M. Verbeek, Mario Mastrangelo, Jennifer Friedman, Tessa Wassenberg, Kathrin Jeltsch, Jan Kulhánek, and Oya Kuseyri Hübschmann. 2020. "Consensus Guideline for the Diagnosis and Treatment of Tetrahydrobiopterin (BH4) Deficiencies." Orphanet Journal of Rare Diseases 15(1):126. doi: 10.1186/s13023-020-01379-8.

Paul, Diane B., and Jeffrey P. Brosco. 2013. The PKU Paradox: A Short History of a Genetic Disease. Baltimore: Johns Hopkins University Press. 
Pijanowska, Dorota, and Elżbieta Remiszewska. 2006. "PH-Based Detection of Phenylalnine by Potentiometric and Colorimetric Methods." Sensors 6(4):428-34. doi: $10.3390 / \mathrm{s} 6040428$.

Robinson, Robert, Liam Wong, Raymond J. Monnat, and Elain Fu. 2016. "Development of a Whole Blood Paper-Based Device for Phenylalanine Detection in the Context of PKU Therapy Monitoring." Micromachines 7(2):28. doi: 10.3390/mi7020028.

Romani, Cristina, Liana Palermo, Anita MacDonald, Ellie Limback, S. Kate Hall, and Tarekegn Geberhiwot. 2017. "The Impact of Phenylalanine Levels on Cognitive Outcomes in Adults with Phenylketonuria: Effects across Tasks and Developmental Stages." Neuropsychology 31(3):242-54. doi: 10.1037/neu0000336.

Tanaka, Shohei, Kenta Adachi, and Suzuko Yamazaki. 2013. "Surface-Enhanced Photochromic Phenomena of Phenylalanine Adsorbed on Tungsten Oxide Nanoparticles: A Novel Approach for 'Label-Free' Colorimetric Sensing." The Analyst 138(9):2536. doi: 10.1039/c3an36650b.

Thiessen, Gregory, Robert Robinson, Kim De Los Reyes, Raymond J. Monnat, and Elain Fu. 2014. "Conversion of a Laboratory-Based Test for Phenylalanine Detection to a Simple Paper-Based Format and Implications for PKU Screening in Low-Resource Settings." Analyst 140(2):609-15. doi: 10.1039/C4AN01627K.

Villalonga, Reynaldo, Akira Fujii, Hiroaki Shinohara, Yasuhisa Asano, Roberto Cao, Shinjiro Tachibana, and Pedro Ortiz. 2007. "Supramolecular-Mediated Immobilization of IPhenylalanine Dehydrogenase on Cyclodextrin-Coated Au Electrodes for Biosensor Applications." Biotechnology Letters 29(3):447-52. doi: 10.1007/s10529-006-9259-4.

Villalonga, Reynaldo, Akira Fujii, Hiroaki Shinohara, Shinjiro Tachibana, and Yasuhisa Asano. 2008. "Covalent Immobilization of Phenylalanine Dehydrogenase on Cellulose Membrane for Biosensor Construction." Sensors and Actuators B: Chemical 129(1):195-99. doi: 10.1016/j.snb.2007.07.097.

van Wegberg, A. M. J., A. MacDonald, K. Ahring, A. Bélanger-Quintana, N. Blau, A. M. Bosch, A. Burlina, J. Campistol, F. Feillet, M. Giżewska, S. C. Huijbregts, S. Kearney, V. Leuzzi, F. Maillot, A. C. Muntau, M. van Rijn, F. Trefz, J. H. Walter, and F. J. van Spronsen. 2017. "The Complete European Guidelines on Phenylketonuria: Diagnosis and Treatment." Orphanet Journal of Rare Diseases 12(1):162. doi: 10.1186/s13023-017-0685-2.

Weiss, David J., Megan Dorris, Amanda Loh, and Laura Peterson. 2007. "Dehydrogenase Based Reagentless Biosensor for Monitoring Phenylketonuria." Biosensors and Bioelectronics 22(11):2436-41. doi: 10.1016/j.bios.2006.09.001.

Wendel, U., Martina Koppelkamm, and W. Hummel. 1991. "Enzymatic Phenylalanine Estimation for the Management of Patients with Phenylketonuria." Clinica Chimica Acta 201(1-2):95-98. doi: 10.1016/0009-8981(91)90029-C.

Wendel, Udo, Werner Hummel, and Ulrich Langenbeck. 1989. “Monitoring of Phenylketonuria: A Colorimetric Method for the Determination of Plasma Phenylalanine Using IPhenylalanine Dehydrogenase." Analytical Biochemistry 180(1):91-94. doi: 10.1016/0003-2697(89)90092-4. 
534 Wu, Ting, Xiaoping Wei, Xionghui Ma, and Jianping Li. 2017. “Amperometric Sensing of LPhenylalanine Using a Gold Electrode Modified with a Metal Organic Framework, a Molecularly Imprinted Polymer, and $\beta$-Cyclodextrin-Functionalized Gold Nanoparticles." Microchimica Acta 184(8):2901-7. doi: 10.1007/s00604-017-2281-5.

Zhuo Wang, Ya-zhu Chen, Su Zhang, and Zhen Zhou. 2005. "Investigation of a PhenylalanineBiosensor System for Phenylketonuria Detection." Pp. 1913-16 in 2005 IEEE Engineering in Medicine and Biology 27th Annual Conference.

541

542 


\section{$543 \quad$ Figure captions}

544 Figure 1: Steps of biosensor fabrication, assay and analysis. Created with BioRender.com

545 Figure 2. Characterisation of biosensor in buffer. A) Michaelis-Menten fit of experimentally 546 determined reaction rates for PDH-mediated L-Phe turnover in buffer. Inset: Calibration plot of 547 [L-Phe] in PBS $1 \mathrm{X} \mathrm{pH} 7.4$ mixed in the measurement buffer. The obtained calibration equation is $548[N A D H] / \sec (\mu M / s)=0.025(1 / s) \cdot[L-P h e](\mu M)+1.807(\mu M / s)$. B) Interference study 549 with plasma optimized conditions $\left(\mathrm{PDH}=3 \mathrm{U} / \mathrm{cm}^{2}, \mathrm{BSA}=28 \mu \mathrm{M}, \mathrm{NAD}{ }^{+}=6 \mathrm{mM}, \mathrm{T}=30^{\circ} \mathrm{C}\right.$, and $550 \mathrm{pH}=10.5)$ Inset: Shelf lifetime of the photometric platform for L-Phe detection measured in the 551 presence of L-Phe $500 \mu \mathrm{M}$.

552 Figure 3. Preclinical validation of the photometric platform for L-Phe sensing on 15 plasma 553 samples. Discrimination between average and high L-Phe values was observed.In figure A) the 554 correlation plot with values provided by the hospital are shown. The obtained calibration 555 equation is $[N A D H] / \sec (\mu M / s)=0.016(1 / s) \cdot[L-P h e]_{\text {Hospital }}(\mu M)+5.419(\mu M / s)$. In 556 figure B) the corresponding analysis, discriminating among the most importan [L-Phe] levels is 557 shown.

558 Figure 4. Preclinical validation of the photometric platform for L-Phe sensing on 30 plasma 559 samples measured in triplicate and 5 whole blood samples. Discrimination between the 3 sets 560 of L-Phe values was observed * stands for $\mathrm{p}<0.05$ and ${ }^{* * *}$ for $\mathrm{p}<0.001$. A) plasma L-Phe values 561 of $300 \mu \mathrm{M}, 450 \mu \mathrm{M}$ and $700 \mu \mathrm{M}$ with results of $t$-test analysis. B) The same methodology as for 562 the the blood plasma was applied for a set of whole blood samples provided by HSJD. The 563 obtained calibration equation is $[N A D H] / \sec (\mu M / s)=0.008(1 / s) \cdot[L-$ Phe $]_{\text {Hospital }}(\mu M)+0.6416(\mu M / s)$. 
Microplate

sensor

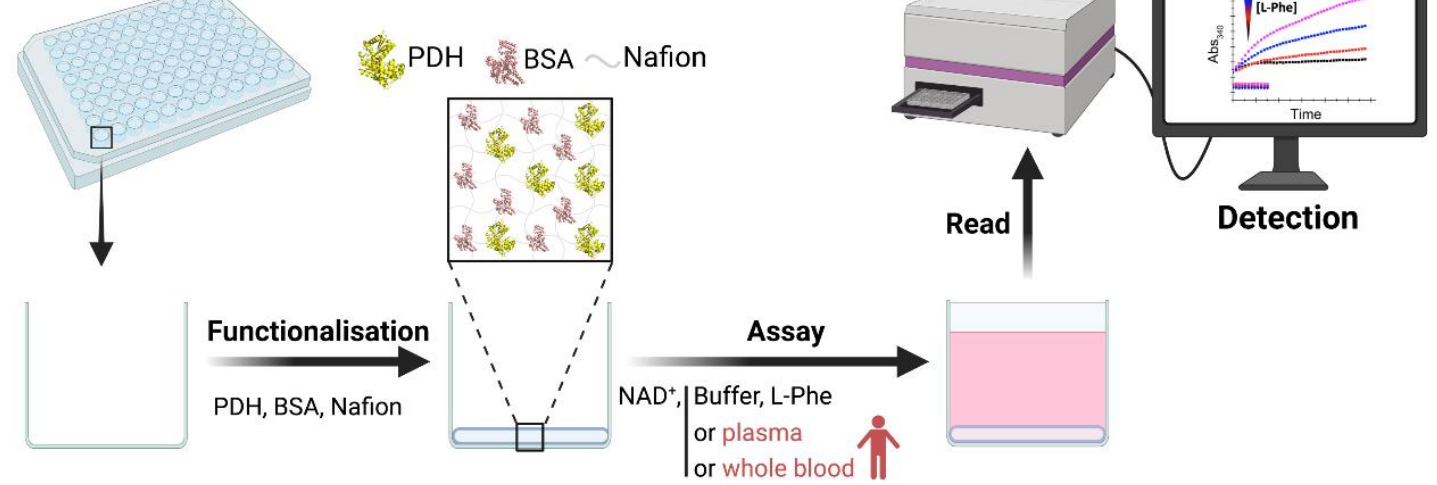

568

569 Figure 1

570 
A

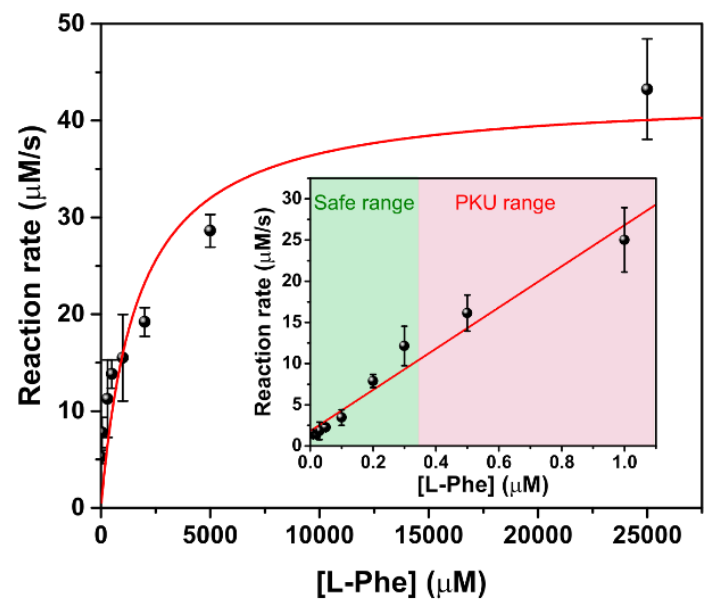

571

\section{$572 \quad$ Figure 2}

573
B

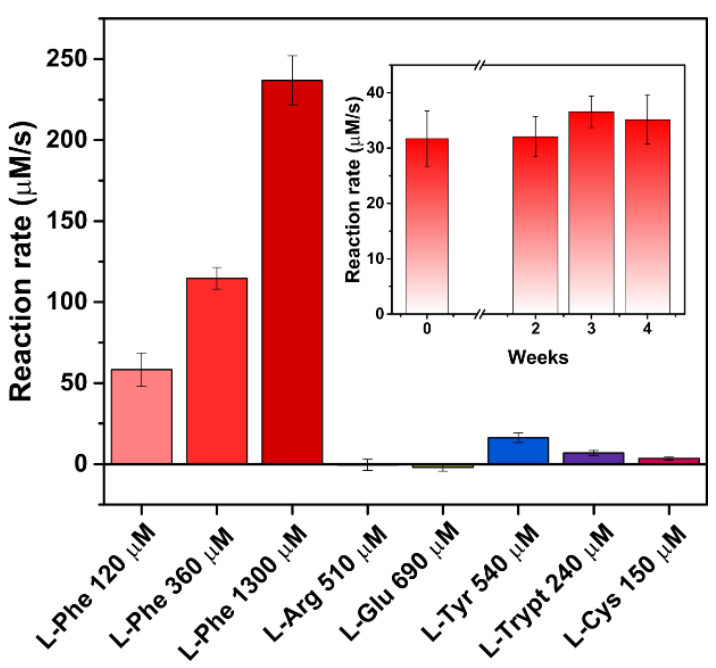


A

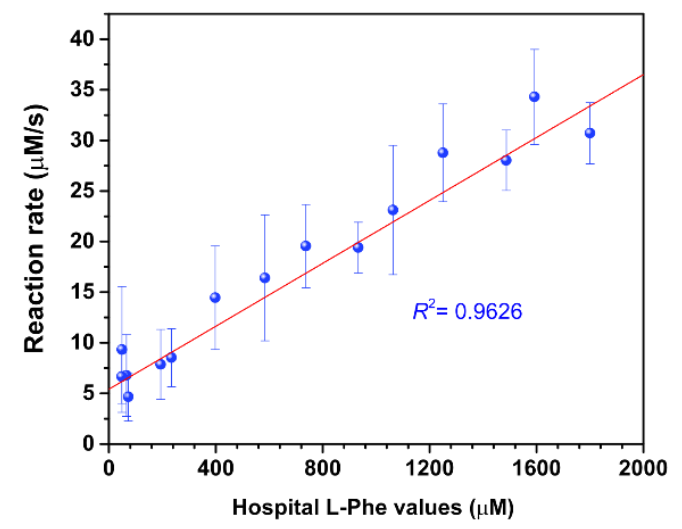

574

$575 \quad$ Figure 3

576
B

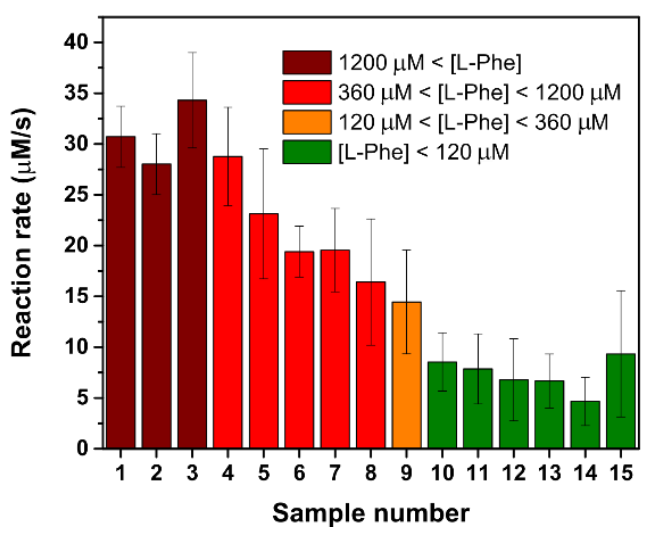


A

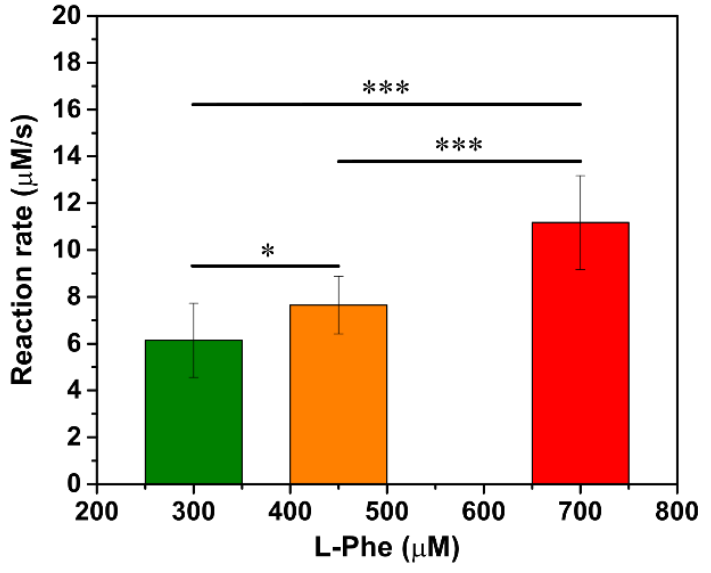

577

$578 \quad$ Figure 4
B

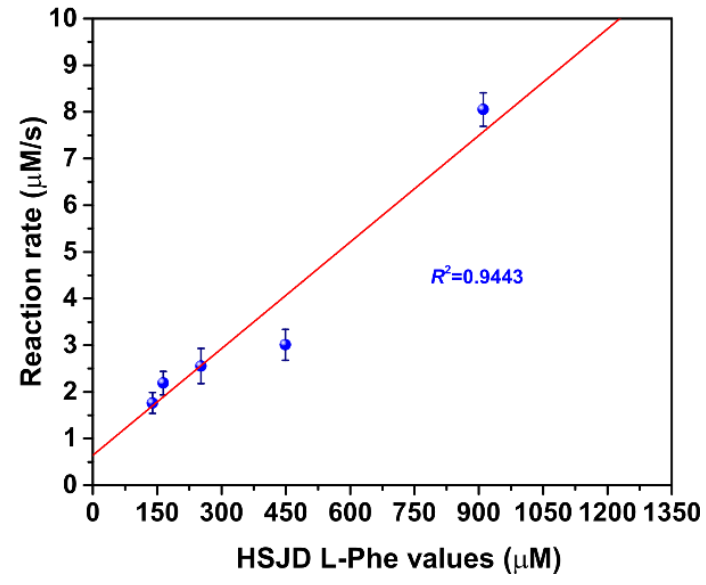

579 


\begin{tabular}{|c|c|c|c|c|c|c|c|c|c|c|c|}
\hline Sensing material & $\begin{array}{l}\text { Detect. } \\
\text { principle }\end{array}$ & $\begin{array}{l}\text { Device } \\
\text { type }\end{array}$ & $\begin{array}{l}\text { LOD } \\
(\mu \mathrm{M})\end{array}$ & $\begin{array}{l}\text { Linear } \\
\text { range } \\
(\mu \mathrm{M})\end{array}$ & $\begin{array}{l}\text { Real } \\
\text { sample }\end{array}$ & Validation & $\begin{array}{l}\text { Temp } \\
\left({ }^{\circ} \mathrm{C}\right)\end{array}$ & Buffer & $\begin{array}{l}\text { Test } \\
\text { time } \\
(\mathrm{min})\end{array}$ & $\begin{array}{l}\text { Interferents } \\
\text { tested }\end{array}$ & Ref \\
\hline $\begin{array}{l}\text { PDH } \\
\text { Brevibacterium-in } \\
\text { liquid }\end{array}$ & $\begin{array}{l}\text { NADH } \\
\text { absorb. } \\
(340 \mathrm{~nm})\end{array}$ & Slope & $10-20$ & $10-300$ & - & $\begin{array}{l}\text { Autom. } \\
\text { AA }\end{array}$ & 30 & $\begin{array}{l}0.1 \quad \mathrm{M} \\
\text { glycine } \\
\text { buffer, } \\
\mathrm{pH} 10.7\end{array}$ & 1 & Tyr, PP & $\begin{array}{l}\text { (Hum } \\
\text { mel et } \\
\text { al. } \\
\text { 1988) }\end{array}$ \\
\hline $\begin{array}{l}\text { PDH Rhodococcus - } \\
\text { in liquid }\end{array}$ & $\begin{array}{l}\text { NADH } \\
\text { absorb. } \\
(340 \mathrm{~nm})\end{array}$ & Endpoint & - & $30-2000$ & Plasma & Autom AA & 21 & $\begin{array}{l}\text { glycine } \\
\text { buffer, } \\
\text { pH } 10.8\end{array}$ & 10 & $P P$, tyr, $A A$ & $\begin{array}{l}\text { (Wen } \\
\text { del, } \\
\text { Kopp } \\
\text { elkam } \\
\text { m, } \\
\text { and } \\
\text { Hum } \\
\text { mel } \\
\text { 1991) }\end{array}$ \\
\hline $\begin{array}{l}\text { PDH Rhodococcus - } \\
\text { in liquid }\end{array}$ & $\begin{array}{l}\mathrm{NADH} \quad-> \\
\text { formazan } \\
(492 \mathrm{~nm})\end{array}$ & kinetic & $1.5-3$ & $30-1200$ & Plasma & $\begin{array}{l}\text { Autom. } \\
\text { AA }\end{array}$ & 21 & $\begin{array}{l}\text { K- } \\
\text { phosph } \\
\text { ate }+ \\
\text { triethan } \\
\text { olamine } \\
+ \text { Triton }\end{array}$ & 30 & $\begin{array}{l}\text { PP, tyr, AA, } \\
\text { pyruvate }\end{array}$ & $\begin{array}{l}\text { (Wen } \\
\text { del, } \\
\text { Hum } \\
\text { mel, } \\
\text { and } \\
\text { Lange } \\
\text { nbeck } \\
\text { 1989) }\end{array}$ \\
\hline $\begin{array}{l}\text { PDH Rhodococcus - } \\
\text { in liquid }\end{array}$ & $\begin{array}{l}\text { NADH } \\
\text { absorb. } \\
(340 \mathrm{~nm})\end{array}$ & Endpoint & - & - & - & $\begin{array}{l}\text { Colorim. } \\
\text { (pH } \\
\text { indicators) }\end{array}$ & RT & $\begin{array}{l}\text { glycine } \\
\text { buffer } \\
\mathrm{pH} \\
10.56\end{array}$ & 90 & - & $\begin{array}{l}\text { (Pijan } \\
\text { owsk } \\
\text { a and } \\
\text { Remis } \\
\text { zewsk } \\
\text { a } \\
\text { 2006) }\end{array}$ \\
\hline $\begin{array}{l}\text { PDH } \\
\text { Thermoactinomyc } \\
\text { es intermedius - } \\
\text { immobilized on } \\
\text { filter membrane }\end{array}$ & $\begin{array}{l}\text { NADH } \\
\text { fluo emiss } \\
(493 \mathrm{~nm})\end{array}$ & slope & $>5$ & $\begin{array}{l}20- \\
10000\end{array}$ & - & - & 25 & $\begin{array}{l}\mathrm{PB}, \\
\mathrm{pH} 8.0 \\
80 \mathrm{mM}\end{array}$ & 5 & $\begin{array}{l}\text { Gly, Tyr, Leu, } \\
\text { Val, Isoleu, } \\
\text { Methio }\end{array}$ & $\begin{array}{l}\text { (Arak } \\
\text { awa } \\
\text { et al. } \\
2011 \text { ) }\end{array}$ \\
\hline $\begin{array}{l}\text { PDH } \\
\text { Sporosarcina } \\
\text { immobilized on UV } \\
\text { transparent well }\end{array}$ & $\begin{array}{l}\text { NADH } \\
\text { absorb. } \\
(340 \mathrm{~nm})\end{array}$ & slope & 1.03 & $30-1000$ & $\begin{array}{l}\text { Plasma, } \\
\text { whole } \\
\text { blood }\end{array}$ & $\begin{array}{l}\text { Plasma, } \\
\text { whole } \\
\text { blood } \\
\text { HPLC }\end{array}$ & 30 & $\begin{array}{l}\text { glycine } \\
\text { buffer } \\
\mathrm{pH} \\
10.56\end{array}$ & 30 & $\begin{array}{l}\text { Arg, D-Trypt, } \\
\text { Tyr, Cys, } \\
\text { Glut }\end{array}$ & $\begin{array}{l}\text { This } \\
\text { work }\end{array}$ \\
\hline I & I & $T$ & 1 & 1 & $T$ & I & $T$ & & & & \\
\hline $\begin{array}{l}\text { PDH Sporosarcina- } \\
\text { in liquid }\end{array}$ & $\begin{array}{l}\mathrm{NADH} \quad-> \\
\text { formazan } \\
(510 \mathrm{~nm})\end{array}$ & slope & $\begin{array}{l}69 \\
\text { (LOQ) }\end{array}$ & $0-9000$ & Plasma & $\begin{array}{l}\text { AAA, } \\
\text { HPLC, } \\
\text { MS/MS }\end{array}$ & - & $\begin{array}{l}\text { K- } \\
\text { phosph } \\
\text { ate }+ \\
\text { triethan } \\
\text { olamine } \\
\text { pH } 8.6\end{array}$ & 1 & Tyr & $\begin{array}{l}\text { (De } \\
\text { Silva, } \\
\text { Oldha } \\
\mathrm{m}, \\
\text { and } \\
\text { May } \\
\text { 2010) }\end{array}$ \\
\hline $\begin{array}{l}\text { PDH - immobilized } \\
\text { on paper }\end{array}$ & $\begin{array}{l}\mathrm{NADH} \quad \rightarrow \\
\text { formazan } \\
(340 \mathrm{~nm})\end{array}$ & Endpoint & 0.3 & $\begin{array}{l}\text { Only } 4 \\
\text { points }\end{array}$ & Plasma & Phe assay & 25 & $\begin{array}{l}50 \mathrm{mM} \\
\text { bis-tris } \\
\text { propan } \\
\text { e pH } 9.3\end{array}$ & $10-20$ & - & $\begin{array}{l}\text { (Thies } \\
\text { sen et } \\
\text { al. } \\
\text { 2014) }\end{array}$ \\
\hline $\begin{array}{l}\text { PDH- immobilized } \\
\text { on paper }\end{array}$ & $\begin{array}{l}\mathrm{NADH} \quad-> \\
\text { formazan } \\
(340 \mathrm{~nm})\end{array}$ & - & - & $60-300$ & Blood & - & 25 & blood & $6+2$ & - & $\begin{array}{l}\text { (Robi } \\
\text { nson } \\
\text { et al. } \\
2016 \text { ) }\end{array}$ \\
\hline
\end{tabular}




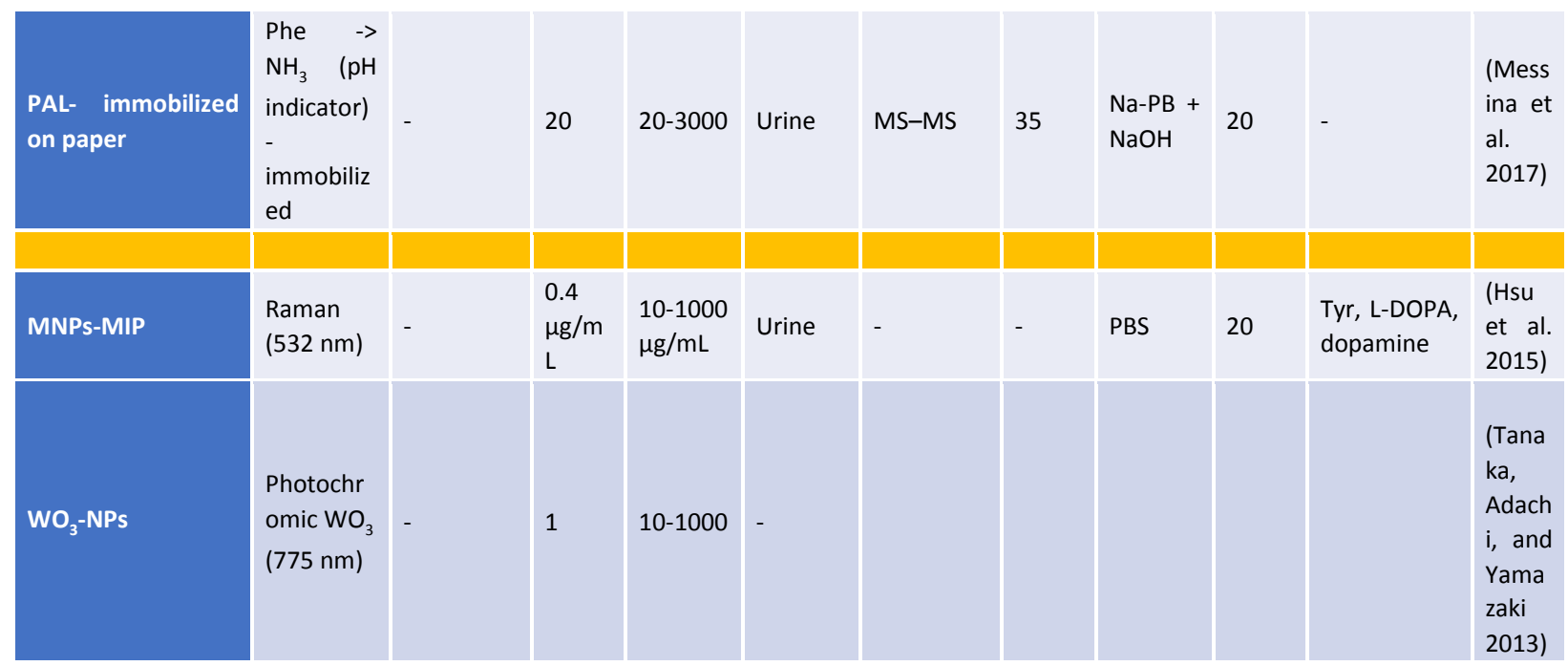

581 Table 1: Comparison with Literature 